\title{
LA FAMILIA Y EL CONSUMO DE ALCOHOL EN ESTUDIANTES UNIVERSITARIOS
}

\section{FAMILY AND ALCOHOL CONSUMPTION IN UNIVERSITY STUDENTS}

\author{
Nora Angélica ArmendáRiz García * \\ María Magdalena Alonso Castillo ${ }^{* *}$ \\ Bertha Alicia Alonso Castillo ${ }^{* * *}$ \\ Manuel Antonio López Cisneros ${ }^{* * * *}$ \\ Linda Azucena Rodríguez Puente ${ }^{* * * *}$ \\ Martha Dalila Méndez Ruiz ${ }^{* * * * *}$
}

\begin{abstract}
RESUMEN
El consumo de alcohol constituye un problema de salud pública por la alta prevalencia del consumo, la mortalidad y trastornos mentales asociados a éste, principalmente en los jóvenes. Objetivo: Identificar la relación e influencia de la historia familiar de consumo de alcohol sobre el consumo de alcohol en los estudiantes universitarios. Material y método: El estudio fue descriptivo, correlacional y predictivo. La población fueron estudiantes universitarios. El muestreo fue aleatorio estratificado con asignación proporcional al tamaño del estrato. Dentro de cada estrato se empleó muestreo aleatorio por conglomerados unietápico. La muestra fueron 680 estudiantes universitarios, poder estadístico del 90\%. Los instrumentos empleados fueron el Inventario de Historia Familiar de Consumo de Alcohol y Cuestionario de Identificación de los Trastornos debidos al Consumo de Alcohol. Resultados: El presentar historia familiar de consumo de alcohol influye de una manera importante en el consumo de alcohol de los estudiantes $(B=0.080, p=0.001)$. Conclusión: La historia familiar de consumo de alcohol influye en el consumo de alcohol de los estudiantes. Se propone un modelo predictivo, el cual en un futuro sirva para la creación de intervenciones de enfermería dirigidas a esta población y sus familias.
\end{abstract}

Palabras clave: Consumo de alcohol, estudiantes, familia, enfermería psiquiátrica.

\footnotetext{
ABSTRACT

Alcohol consumption is considered a public health problem due to the high use prevalence, mortality and mental disorders associated, particularly in young people. Objective: To identify family history consumption

* Enfermera, Profesora Facultad de Enfermería, Universidad Autónoma de Nuevo León, México. Correo electrónico: nordariz@hotmail.com

${ }^{* *}$ Enfermera. Profesora Facultad de Enfermería, Universidad Autónoma de Nuevo León, México. Correo electrónico: magdalena_alonso@hotmail.com

${ }^{* * *}$ Enfermera. Profesora Facultad de Enfermería, Universidad Autónoma de Nuevo León, México. Correo electrónico: balonso_mx@yahoo.com.mx

${ }^{* * * *}$ Enfermero. Profesor DES Ciencias de la Salud, Universidad Autónoma del Carmen, Campeche, México. Correo electrónico: mlcisneros@hotmail.com

${ }^{* * * * *}$ Enfermera. Estudiante de Doctorado en Ciencias de Enfermería, Facultad de Enfermería, Universidad Autónoma de Nuevo León, México. Correo electrónico: lrpuente88@hotmail.com

${ }^{* * * * * *}$ Enfermera. Estudiante de Doctorado en Ciencias de Enfermería, Facultad de Enfermería, Universidad Autónoma de Nuevo León, México. Correo electrónico: estrellita_lilet@hotmail.com
} 
and its relation and influence on alcohol consumption in students. Methods: The study was descriptive, correlational and predictive. The population were university students. Stratified random sampling allocation was proportional to the size of the stratum. Within each stratum was used one-stage random cluster sampling. The sample was composed of 680 college students, with $90 \%$ statistical power. Instruments used were the Family History Inventory of Alcohol Consumption and Disorder Identification Questionnaire due to Alcohol Consumption. Results: The present family history of alcohol consumption influenced in a major way the consumption of alcohol by students $(B=0.080, p=0.001)$. Conclusions: Family history of alcohol consumption influences alcohol consumption of students. The contribution of this study is to propose a predictive model which in the future will help in the creation of nursing interventions aimed at this population and their families.

Key words: Alcohol consumption, students, family, psychiatric nursing.

Fecha recepción: 17/12/13. Fecha aceptación: 18/12/14.

\section{INTRODUCCIÓN}

El consumo de alcohol cumple una función social, muchos jóvenes ingresan en el mundo de las sustancias psicoactivas precisamente por este motivo, es una manera de sentir y pensar en base a algunas premisas socioculturales del grupo al que ellos pertenecen. La ingesta y abuso de alcohol en los jóvenes ha adquirido dimensiones importantes en nuestra sociedad, pasando a constituir una de las mayores preocupaciones en el ámbito de la salud, debido a que se observan estadísticas alarmantes respecto a la cantidad y prevalencia de consumo de alcohol en los jóvenes (1, 2).

Respecto a los Años de Vida Ajustados por Discapacidad (AVISA) por causa de la ingesta excesiva de alcohol a nivel mundial, se reporta que en la población masculina es el causante del $7.4 \%$ de los años de vida ajustados por discapacidad y el $1.4 \%$ en la población femenina, por lo que se señala como el tercer factor de enfermedad y discapacidad a nivel global (3). Respecto a la mortalidad, la Organización Panamericana de la Salud (OPS) (4) señala que el abuso de alcohol es el responsable de 2.5 millones de muertes por año en el mundo, presentándose en mayor porcentaje en hombres $(6.2 \%)$ que en mujeres $(1.1 \%)$, e incluye a nivel mundial jóvenes de entre 15 y 29 años (320 mil) que mueren anualmente por esta causa; lo que representa el $9 \%$ de todas las muertes en este sector de la población.

En México, las enfermedades no transmisibles ocupan el primer lugar de AVISA perdidos, la ingesta y abuso de alcohol se considera parte de éstas y específicamente la ingesta es el responsable del $4.8 \%$ de años de vida saludables perdidos. Así mismo, se ha reportado un aumento paulatino en el consumo de alcohol, especialmente entre la población joven. La Encuesta Nacional de Adicciones (5), realizada el 2002 mostró que el 72.2\% de los hombres y el $42.7 \%$ de las mujeres en la población reportaron consumir alcohol en el último año, mientras que los resultados de la encuesta aplicada el 2008 mostró un aumento del $6.8 \%$ en hombres y un $10.3 \%$ en las mujeres $(5,6)$.

También se ha identificado que casi $27 \mathrm{mi}$ llones de mexicanos entre 12 y 65 años beben alcohol con un patrón típico de grandes cantidades de alcohol por ocasión de consumo y con frecuencias de menos de una vez por mes. El consumo de cinco copas o más considerado excesivo para los hombres y de cuatro copas o más para las mujeres, al menos una vez a la semana, es mayor entre hombres que entre mujeres, en una proporción de 5.8 hombres por cada mujer. 
Un aspecto importante a considerar es que la primera ocasión que los adolescentes beben alcohol casi nunca es el resultado de una decisión personal, sino que suele ser la respuesta a una invitación o presión de algunos miembros de la sociedad, los cuales pueden ser familiares. En este sentido la experiencia por parte del joven suele ser positiva porque le habrá servido para identificarse y ser uno más del grupo, o bien liberarse de la presión social. Este resultado positivo favorece el tránsito de un consumo experimental, a la ingesta frecuente de esta sustancia, especialmente si esta práctica es habitual en el grupo, para posteriormente consolidarse como un hábito o estilo de vida. Como se puede observar, la ingesta de alcohol es parte de la vida social, dado que está presente como vehículo de socialización, en festividades con arraigo cultural, como parte de los acuerdos laborales, sin embargo la forma en que los jóvenes inician y mantienen esta conducta es una preocupación en salud que forma parte de las agendas del sector salud en México.

Las consecuencias negativas derivadas del abuso de alcohol suelen referirse a alteraciones de las relaciones con la familia, compañeros y maestros, bajo rendimiento escolar, agresiones, violencia, alteraciones del orden público y conductas de alto riesgo, como conducir bajo la influencia alcohólica, así como actividades sexuales de riesgo.

Se desconoce qué jóvenes continuarán abusando del alcohol durante la edad adulta, y cuáles abandonarán el abuso sin necesidad de intervención o tratamiento. No obstante se deben valorar los factores de riesgo, en particular los antecedentes familiares de dependencia o ingesta de alcohol u otros psicotrópicos en la familia, la permisibilidad del consumo de alcohol en los integrantes de menor edad dentro de la familia. Una historia familiar de consumo de alcohol positiva y permisible predispone a que los jóvenes presenten una conducta de manera frecuente y en algunos casos esta conducta se presenta de manera dañina o dependiente (7).
Tanto en hombres como en mujeres, el grupo de edad que muestra los niveles más altos de ingesta de esta sustancia es de 18 a 29 años. Los resultados indican que la población adolescente y de jóvenes está reproduciendo los patrones de consumo de la población adulta donde estas diferencias en cantidad y frecuencia de la ingesta de alcohol se reducen rápidamente, especialmente entre jóvenes estudiantes de grandes ciudades debido a los cambios en el contexto sociocultural (1).

Sin embargo, en la población de estudiantes universitarios existen escasos estudios en los cuales se trate de identificar cómo la familia se relaciona o influye en la ingesta de alcohol de esta población. Es importante mencionar que en la cultura mexicana el estudiante universitario permanece como miembro del núcleo familiar generalmente hasta que decide vivir en pareja o se gradúa y es capaz de independizarse económicamente; por ese motivo es la importancia de considerar el antecedente o la historia de consumo de alcohol de la familia en el presente estudio, en comparación con otras culturas en las cuales el joven universitario emigra del hogar a edades tempranas.

La influencia de la familia incluidos los padres y hermanos continúa siendo importante para el desarrollo y estructura firme de la personalidad, y del aprendizaje de mecanismos de afrontamiento y de estrategias de asertividad de los estudiantes universitarios. Si entre los miembros de la familia se presentan conductas de ingesta de alcohol, éste puede ser un factor que incremente la probabilidad de beber alcohol en el estudiante universitario, ya que en el núcleo familiar esta conducta es permitida y aprendida (7-10). Sin embargo, también se ha observado que tener familiares que muestren problemas de ingesta de alcohol, aunado a la presencia de dificultades familiares por esta causa, puede ser un factor determinante del deseo de no beber alcohol dado a que han vivido o experimentado las consecuencias negativas del alcohol en sus familias o en seres queridos. 
Algunos autores en estudios (11) realizados en adolescentes y jóvenes muestran relaciones significativas entre la historia familiar de consumo de alcohol por parte de los padres y el consumo de los estudiantes. Así mismo Villarreal et al. (12) propusieron un modelo socio-comunitario con el objetivo de analizar las relaciones existentes entre variables familiares con el consumo de alcohol en adolescentes, observándose que la ingesta de alcohol se correlaciona de forma positiva con el consumo de la familia y amigos, y a través del modelo estructural se observó que el consumo de alcohol está relacionado positivamente con el consumo de la familia. En un estudio realizado en universitarios en Colombia (13) se identificó que quienes tienen padres que se emborrachan presentan en mayor proporción consumo de alcohol riesgoso a diferencia de los jóvenes que sus padres no se emborrachan. A pesar de la revisión de la literatura no se localizaron artículos de investigación realizados en estudiantes universitarios donde se considerara la relación y efecto del consumo de alcohol de ambos padres y de los hermanos sobre la ingesta de alcohol de los jóvenes.

Desde la perspectiva holística de enfermería como disciplina del cuidado se ha determinado la necesidad de considerar a la familia y sus comportamientos como factor de riesgo o de protección en la adopción de hábitos saludables en los adolescentes y jóvenes.

Por ese motivo el profesional de enfermería debe identificar cómo las conductas y hábitos al interior de la familia se relacionan e influyen en la ingesta de alcohol de sus miembros, especialmente en los hijos. La información obtenida de este fenómeno será útil en el cuidado de promoción de la salud y de prevención del uso y abuso de alcohol en este grupo prioritario del sector salud.

Por tal motivo, el objetivo general del presente estudio es identificar la relación e influencia de la historia familiar de consumo de alcohol sobre el consumo de alcohol en los estudiantes universitarios.

\section{MATERIAL Y MÉTODO}

El diseño del presente estudio fue descriptivo y correlacional. En una población de 34.509 jóvenes universitarios de una universidad pública del Estado de Nuevo León, México. La muestra del estudio se conformó por 680 estudiantes universitarios. El muestreo fue aleatorio estratificado con asignación proporcional al tamaño del estrato. El cálculo de la muestra se realizó a través del paquete estadístico n'Query Advisor Versión 4.0 ® (14), para calcular la muestra se utilizó un nivel de significancia de .05, con un Coeficiente de Determinación de $\mathrm{R}^{2}=.09$ y un poder estadístico del $90 \%$ para un Modelo de Regresión Lineal Múltiple, y una tasa de no respuesta del 15\%.

Las mediciones se llevaron a cabo mediante la Cédula de Datos Personales y de Prevalencia de Consumo de Alcohol (CDPPCA) del participante, así como un instrumento que mide la Historia Familiar de Consumo de Alcohol y el consumo de alcohol de los estudiantes universitarios fue medido a través del Cuestionario de Identificación de los Trastornos debidos al Consumo de Alcohol (AUDIT).

La CDPPCA se divide en dos secciones, una relacionada con aspectos personales y personas con las que vive y que consumen alcohol; y la segunda sección se enfoca en la ingesta de alcohol respecto a sus prevalencias alguna vez en la vida, en el último año y el último mes, así como información relacionada a la cantidad de bebidas alcohólicas que consuman los miembros de la familia.

Se utilizó el Inventario de Historia Familiar de Consumo de Alcohol [IHFCA] (8) para identificar esta variable. Estas preguntas indagan la frecuencia de consumo de alcohol del padre y madre en términos de frecuencia de consumo, embriaguez, así como la forma de consumo en términos de si los padres consumen alcohol solos o juntos. También se cuestiona si el adolescente está presente 
cuando alguno sus padres consumía alcohol. Estas preguntas se evaluaron cada una por separado y las preguntas se calificaron con puntajes de cero a seis y de cero a dos en una sola pregunta, si la respuesta es cero se considera historia familiar negativa, y de uno a seis se considera una historia familiar positiva al consumo de alcohol. Lo que significa que se considera historia familiar de consumo negativa cuando el entrevistado notificó haber vivido con padres abstemios o cuyo consumo era ocasional, mientras que la historia familiar de consumo positiva se determinó con base en los reportes de consumo frecuente y consuetudinario de los padres. Este cuestionario reportó un Alpha de Cronbach de 0.79 en población adulta en México por Natera et al. (8), en el presente estudio se presentó un coeficiente de confiabilidad de 0.80 .

Para evaluar la ingesta de alcohol en los estudiantes universitarios se empleo el Cuestionario de Identificación de los Trastornos debidos al Consumo de Alcohol [AUDIT por sus siglas en Inglés] (15), que fue desarrollado por la Organización Mundial de la Salud (OMS), como un método para examinar el consumo de alcohol en atención primaria. Por su parte, De la Fuente y Kershenobich (16) validaron el instrumento en población mexicana obteniendo una sensibilidad de $80 \%$ y una especificidad de $89 \%$. Este instrumento ayuda a identificar el consumo de alcohol sensato o de bajo riesgo, dependiente o riesgoso y el consumo excesivo o dañino (16, 17).

El AUDIT tiene un valor mínimo de 0 y máximo de 40 puntos. La clasificación del patrón de consumo se realiza sumando el total de los reactivos y los puntos de corte son: de 0 a 3 puntos se considera consumo sensato o sin riesgo, de 4 a 7 puntos indica un consumo dependiente y de 8 puntos a más sugiere consumo de alcohol dañino. Este instrumento ha sido empleado en mujeres y adultos, mostrando una consistencia interna aceptable con Alpha de Cronbach de 0.89$0.93(18-20)$.
Para iniciar con la implementación del presente estudio se requirió de la aprobación de la Comisión de Investigación y la Comisión de Ética de la Facultad de Enfermería de la Universidad Autónoma de Nuevo León con número de registro de FAEN-D-807. Se solicitó autorización por escrito a las autoridades de las facultades de la universidad pública, donde se llevó a cabo el presente estudio. Una vez obtenido el permiso para la realización del estudio en cada facultad se solicitaron las listas de alumnos inscritos en el ciclo escolar agosto-diciembre 2011, con información del número total de alumnos de la facultad, una vez obtenida esta información, se calculó la muestra. La selección de los grupos participantes por semestre fue aleatoria a través de tabla de números aleatorios conforme a las listas obtenidas.

En el caso de los participantes adultos, el consentimiento informado se les entregó para que lo leyeran y en el caso de ser menor de edad se le entregó un consentimiento informado para padres y un asentimiento para el alumno con el objetivo que los padres autorizaran o negaran la participación de su hijo(a) en el proyecto de investigación. Para la aplicación de los instrumentos se consideró que no existieran distractores en el aula donde se aplicaron los cuestionarios. La aplicación de éstos estuvo a cargo de dos encuestadores previamente capacitados. Al terminar el llenado de los instrumentos se les solicitó a los alumnos que introdujeran los instrumentos contestados dentro de un sobre sin ningún tipo de identificación y luego depositaron el sobre en una caja, la cual se encontraba al finalizar el salón de clases con el propósito de preservar el anonimato y la confidencialidad; en todo momento se consideraron los aspectos relativos a la Ley General de Salud en materia de Investigación. Finalmente se agradeció la participación de los estudiantes.

Para analizar los datos del estudio se utilizó el paquete estadístico Statistical Package for the Social Sciences (SPSS) versión 17.0 para Windows. Se empleó estadística 
descriptiva e inferencial. La estadística descriptiva permitió conocer las características de los participantes del estudio a través de frecuencias, proporciones, medidas de tendencia central así como medidas de variabilidad. Posteriormente se utilizó la prueba de normalidad Kolmogorov-Smirnov con corrección de Lilliefors para decidir el uso de pruebas paramétricas o no paramétricas; en virtud de que las variables numéricas no mostraron normalidad se decidió utilizar estadística no paramétrica o de libre distribución.

Para el objetivo que consiste en determinar la relación y efecto de la historia familiar de consumo de alcohol sobre el consumo de alcohol en los estudiantes universitarios, se empleó el Coeficiente de Correlación de Spearman y Regresión Múltiple.

\section{RESULTADOS}

Respecto a los datos sociodemográficos de los participantes se observó que la mayoría de los estudiantes se encontraron entre los 17 y 20 años de edad (59.7\%). Los estudiantes en mayor proporción fueron hombres (57.4\%) y el $42.6 \%$ son mujeres, así mismo el $97.6 \%$ no viven en pareja. Los participantes presentan un promedio de $20.2(\mathrm{DE}=2.56)$ años de edad. En la prevalencia de consumo de alcohol se observó que el 92.2\%, IC 95\% [90-94] de los participantes ha consumido alcohol alguna vez en su vida, de los cuales el $85.1 \%$ IC 95\% [82-88] ha consumido en el último año, en el último mes el $60.4 \%$ IC 95\% [57-64] de ellos ha consumido alcohol y en la última semana el 40.1\%, IC 95\% [3644] de los estudiantes ha consumido alcohol. No obstante el 7.8\%, IC 95\% [5-9] de los participantes nunca ha consumido alcohol.

Respecto a la cantidad de bebidas alcohólicas que ingieren en un día típico presentaron una media de $4.3(\mathrm{DE}=4.0)$ bebidas. También se reportó que la sumatoria del inventario de historia familiar de consumo mostró la presencia de una media de 14.13 $(\mathrm{DE}=11.14)$ antecedentes de consumo en la familia, lo que indica que se presenta historia familiar de consumo de alcohol positiva en los estudiantes universitarios.

Así mismo se observó que los estudiantes que consumen alcohol, en mayor proporción, presentan consumo sensato $(45.2 \%$, IC 95\% [41-99]) seguido por el consumo riesgoso o dependiente (28.6\%, IC 95\% [24-32]) y dañino o perjudicial (26.2\%, IC 95\% [22-29]).

Aunado a lo anterior se identificó que respecto al sexo por prevalencias de consumo de alcohol los hombres presentan mayor porcentaje en la prevalencia de consumo de alcohol alguna vez en la vida $\left(\chi^{2}=3.99, p=\right.$ $.046)$, en el último año $\left(\chi^{2}=5.67, p=.017\right)$, en el último mes $\left(\chi^{2}=21.55, p=.001\right)$, y en la última semana $\left(\chi^{2}=40.89, p=.001\right)$, a diferencia de las mujeres que presentan menor prevalencia de consumo. La mujeres reportaron en mayor proporción nunca haber consumido alcohol (10.3\%) respecto a los varones.

En relación a la historia familiar (Tabla 1) también se identificaron diferencias significativas de consumo de alcohol respecto a la historia familiar de consumo ( $\mathrm{U}=21947.50$, $p=.001$ ), observándose que los estudiantes universitarios que presentan antecedentes familiares de consumo presentan puntajes de consumo de alcohol más altos $(\bar{X}=5.93, \mathrm{Mdn}$ $=5.00, \mathrm{DE}=5.23)$ a diferencia de los estudiantes que no presentan dichos antecedentes $(\bar{X}=4.39, \mathrm{Mdn}=2.50, \mathrm{DE}=4.63)$.

Los resultados que responden a lo establecido en el objetivo del presente estudio muestran que la historia familiar de consumo de alcohol $\left(\mathrm{r}_{\mathrm{s}}=.285, \mathrm{p}<.01\right)$, se relaciona positivamente con el consumo de alcohol del estudiante universitario, lo que indica que la presencia de historia familiar de consumo de alcohol mayor es la ingesta de consumo de esta sustancia por parte de los estudiantes. Así mismo respecto a la influencia de la historia familiar de consumo de alcohol y el consumo 
de alcohol de los estudiantes universitarios, sumo de alcohol y el sexo influye $\left(\mathrm{R}^{2}=9.5 \%\right.$, se observó que existe un efecto significativo, $p=0.001$ ) en el consumo de alcohol en los esel cual indica que la historia familiar de con- tudiantes universitarios (Tabla 2).

Tabla1. Consumo de alcohol (AUDIT) de acuerdo a la historia familiar de consumo de alcohol.

\begin{tabular}{lcccccc}
\hline & \multicolumn{7}{c}{ Consumo de alcohol (AUDIT) } \\
\hline $\begin{array}{l}\text { Historia familiar de consumo } \\
\text { de alcohol }\end{array}$ & $\mathrm{n}_{2}$ & $\overline{\mathrm{X}}$ & $\mathrm{Mdn}$ & $\mathrm{DE}$ & $\mathrm{U}$ & $\mathrm{p}$ \\
\hline Sin antecedentes & 120 & 4,39 & 2,5 & 4,63 & 21947,5 & 0,001 \\
Con antecedentes & 459 & 5,93 & 5 & 5,23 & & \\
\hline
\end{tabular}

Nota: $A U D I T=$ Cuestionario de identificación de los trastornos debidos al consumo de alcohol, $n_{2}=$ participantes que beben alcohol.

$\bar{X}=$ media, Mdn= Mediana, DE= Desviación estándar, $U=D E$ Mann Whitney, $p=$ Significancia Estadística.

Tabla 2. Influencia de la historia familiar de consumo de alcohol y el consumo de alcohol de los estudiantes universitarios

\begin{tabular}{|c|c|c|c|c|c|c|}
\hline Fuente de variación & $S C$ & \multicolumn{2}{|c|}{$d f$} & $C M$ & $F$ & $p$ \\
\hline Regresión & 463,3 & \multicolumn{2}{|c|}{1} & 463,3 & 17,95 & 0,001 \\
\hline Residual & 14887,8 & \multicolumn{2}{|c|}{577} & 25,8 & & \\
\hline \multicolumn{7}{|l|}{$R^{2}=9.5 \%$} \\
\hline \multirow{2}{*}{$\begin{array}{l}\text { Modelo } \\
\left(n_{2}=579\right)\end{array}$} & \multicolumn{3}{|c|}{ Coeficientes } & \multicolumn{3}{|c|}{ Sig } \\
\hline & $B$ & $E E$ & $ß$ & $t$ & & $p$ \\
\hline (Constante) & 4,41 & 0,353 & - & 12,5 & & 0,001 \\
\hline $\begin{array}{l}\text { Historia familiar de } \\
\text { consumo de alcohol }\end{array}$ & 0,08 & 0,019 & 0,174 & 4,23 & & 0,001 \\
\hline Sexo & $-2,97$ & 0,422 & $-0,283$ & $-7,01$ & & 0,001 \\
\hline
\end{tabular}

Nota: AUDIT = Cuestionario de identificación de los trastornos debidos al consumo de alcohol, IHFC= Inventario Historia Familiar de Consumo de Alcohol.

\section{DISCUSIÓN Y CONCLUSIÓN}

Se observó que aquellos estudiantes universitarios que presentaban antecedentes de consumo de alcohol por parte de algún familiar cercano fueron quienes presentaron una me- dia más alta de consumo de alcohol a diferencia de los estudiantes que no presentaron este antecedente. Estos resultados son concordantes con otros estudios que mostraron que los estudiantes que tenían antecedentes familiares de consumo de alcohol consumían en mayor cantidad que aquellos que no 
presentaron este antecedente familiar $(7,13$, 21). Estos resultados pueden ser explicados por la socialización familiar; en este núcleo las personas se convierten en seres sociales, y de esta manera interiorizan las normas, valores y conductas que dan sentido social a las relaciones, de esta manera si en la familia se practica la conducta de consumo de alcohol, puede ser que ésta se convierta en un factor decisivo para que el consumo de alcohol se presente en los jóvenes estudiantes que son miembros de estas familias como vehículo de socialización $(8-10,22)$.

Otros estudios muestran que la familia puede ser un factor de protección para el consumo de drogas, siempre y cuando en ella se lleven a cabo conductas saludables y donde el vínculo familiar es fuerte; sin embargo existen estudios que evidencian que la presencia de consumo de alcohol, principalmente en los padres, puede tener en los hijos influencia importante para que ellos presenten y mantengan una conducta de consumo de alcohol en la juventud y vida adulta, esto posiblemente porque esta conducta es aprendida y permitida al interior del núcleo familiar y así se reproduzca culturalmente como forma de vida y fortalecimiento de sentimiento de pertenencia $(2,6,11,23)$, lo cual provoca en los jóvenes que conceptualicen el consumo de alcohol como una conducta normal, que puede pasar desde un consumo sensato hasta un consumo dependiente o dañino $(6,11)$.

Se concluye que la familia en los estudiantes universitarios es una influencia muy importante, la cual puede favorecer la presencia de estilos de vida saludables; sin embargo si en la familia se practican estilos de vida no saludables como el consumo de alcohol, esta práctica influirá en los miembros de ésta, especialmente en los jóvenes que comenzaran con la adopción de patrones que aprenden desde su primer círculo de socialización como es la familia y posteriormente ellos en la universidad posiblemente se encontrarán con compañeros en cuyas familias también se permite el consumo de alcohol, y por ende ésta será una práctica común en la vida del estudiante universitario.

Es importante destacar que los resultados del presente estudio aportan para el diseño e implementación de futuras intervenciones de enfermería o multiprofesionales que podrían enfocarse en la evidencia científica obtenida en el presente estudio que incluiría el manejo de formas de afrontamiento saludables para reducir el consumo de alcohol, la orientación a los padres desde el ciclo de vida familiar, donde construyen el futuro de sus hijos, el trabajar con los jóvenes sobre sus habilidades sociales y sus valores positivos podrían ser elementos a considerar en protocolos de cuidado preventivo de la conducta de consumo de alcohol. Sin duda la meta del cuidado de enfermería es mantener un estado de bienestar de las personas y sus familias, el reducir factores de riesgo de comportamientos no saludables como el uso y abuso de alcohol.

\section{REFERENCIAS}

1. Consejo Nacional Contra las Adicciones (Mex). Encuesta Nacional de Adicciones 2008 [Internet]. México: D.R. (C) Instituto Nacional de Salud Pública; 2008 [citado 13 jun 2013]. 173 p. Disponible en: http:// www.conadic.salud.gob.mx/pdfs/ena08/ ENA08_NACIONAL.pdf

2. Armendáriz NA, Villar MA, Alonso MM, Alonso BA, Oliva NN. Eventos estresantes y su relación con el consumo de alcohol en estudiantes universitarios. Investig. Enferm. Imagen Desarr. 2012; 14(2): 97-112.

3. Organización Panamericana de la Salud (OPS). Alcohol, género, cultura y daños en las Américas: Reporte final del estudio multicéntrico OPS [Internet]. Washington D.C: OPS; 2007 [citado 18 ene 2013]. 70 p. Disponible en: http://www1.paho. org/Spanish/DD/PIN/Multicentrico_Espa\%C3\%B1ol.pdf?ua=1 
4. Organización Mundial de la Salud (OMS). International Statistical Classification of Diseases and Related Health Problems 10th Revision (ICD-10 Version: 2010) [Internet]. Washington D.C: OMS; 2010 [citado 18 feb 2013]. Disponible en: http://apps.who.int/classifications/apps/ icd/icd10online/.

5. Consejo Nacional Contra las Adicciones (Mex). Encuesta Nacional de Adicciones 2002 [Internet]. México: D.R. (C) Instituto Nacional de Salud Pública; 2002 [citado 7 ene 2013]. 222 p. Disponible en: http:// www.uade.inpsiquiatria.edu.mx/pagina contenidos/libros/ena_2002_resultados. pdf

6. Lozano R, Marina-Franco F, Solís P. El peso de la enfermedad crónica en México: retos y desafíos ante la creciente epidemia. Salud Pública Mex. 2007; 49: 283287.

7. Brook J, Balka E, Crossman A, Dermatis H, Galanter M, Brook D. The Relationship between Parental Alcohol Use, Early and Late Adolescent Alcohol Use, and Young Adult Psychological Symptoms: A Longitudinal Study. Am J Addict. 2010; 19(6): 534-542.

8. Natera G, Borges G, Medina MA, Solís L, Tiburcio M. La Influencia de la historia familiar de consumo de alcohol en hombres y mujeres. Salud Pública Mex. 2001; 43(1): 17-26.

9. Musito G, Jiménez T, Murgui S. Funcionamiento familiar, autoestima y consumo de sustancias en adolescentes: Un modelo de mediación. Salud Pública Mex. 2007; 49(1): 3-10.

10. Mota N, Álvarez-Gil R, Corral M, Rodríguez HS, Parada M, Crego A, et al. Risky alcohol use and heavy episodic drinking among Spanish University students: a two-year follow-up. Gac Sanit. 2010; 24(5): 372-377.

11. Landero R, Villarreal ME. Consumo de alcohol en estudiantes, en relación con el consumo familiar y de los amigos. Psico- logía y Salud. 2007; 17(1): 17-23.

12. Villarreal M, Sánchez J, Musitu G, Varela R. El Consumo de Alcohol en Adolescentes Escolarizados: Propuesta de un Modelo Sociocomunitario. Intervención Psicosocial. 2010; 19(3): 253-264.

13. Lema L, Varela M, Duarte C, Bonilla M. Influencia familiar y social en el consumo de alcohol en jóvenes universitarios. Rev. Fac. Nac. Salud Pública. 2011; 29(3): 264271.

14. Elashoff DJ, Dixon JW, Fathenringham N. n'Query Advisor ${ }^{\circledR}$ copyright [software de computadora] E.U.: Statistical Solutions Ltd, 1997.

15. Babor TF, Higgins-Biddle JC, Saunders J, Monteiro M. AUDIT. Cuestionario de identificación de los trastornos debido al consumo de alcohol [Internet]. Washington D.C: OMS; 2001 [citado 21 ene 2013]. 40 p Disponible en: http://www.who.int/ substance_abuse/activities/en/AUDITmanualSpanish.pdf

16. De la Fuente J, Kershenobich D. El alcoholismo como problema médico. Revista de Invest. Clin. UNAM. 1992; 35(2): 4751.

17. Morales J, Fernández I, Tudón H, Escobedo J, Zárate A, Madrazo M. Prevalencia de consumo riesgoso y dañino de alcohol en derechohabientes del Instituto Mexicano del Seguro Social. Salud Pública Mex. 2002; 44(2): 113-121.

18. Alonso MM. Hacia la construcción de un modelo explicativo de factores de riesgo y consumo de alcohol en mujeres adultas [Tesis doctoral]. Monterrey, Nuevo León, México: Facultad de Psicología de la Universidad Autónoma de Nuevo León; 2008. $221 \mathrm{p}$.

19. Alvarado M, Garmendia M, Acuña G, Santis R, Arteaga O. Validez y confiabilidad de la versión chilena del Alcohol Use Disorders Identification Test (AUDIT). Rev Med Chil. 2009; 137(11): 1463-8.

20. Pérula LA, Fernández J, Arias R, Muriel M, Márquez E, Ruiz R. Validación del 
cuestionario AUDIT para la identificación del consumo de riesgo y de los trastornos por el uso de alcohol en mujeres. Aten Primaria. 2005; 36(9): 499-506.

21. Salazar IC, Arrivillaga M. El consumo de alcohol, tabaco y otras drogas, como parte del estilo de vida de jóvenes universitarios. Revista Colombiana de Psicología. 2004; 13: 74-89.
22. Pons J, Buelga S. Factores Asociados al Consumo Juvenil de Alcohol: Una Revisión desde una Perspectiva Psicosocial y Ecológica. Psycho Inter. 2011; 20(1): 7594.

23. Dantzer C, Wardle J, Fuller R. et al. International study of heavy drinking: Attitudes and sociodemographic factors in university students. J Am Coll Health. 2006; 55(2): 83-89. 